\title{
A NUMERICAL ALGORITHM FOR SINGULAR MULTI-POINT BVPS USING THE REPRODUCING KERNEL METHOD
}

\author{
YUNTAO Jia ${ }^{\mathrm{a}, *}$ AND YingZHEN Lin ${ }^{\mathrm{b}}$
}

\begin{abstract}
In this paper, we construct a complex reproducing kernel space for singular multi-point BVPs, and skillfully obtain reproducing kernel expressions. Then, we transform the problem into an equivalent operator equation, and give a numerical algorithm to provide the approximate solution. The uniform convergence of this algorithm is proved, and complexity analysis is done. Lastly, we show the validity and feasibility of the numerical algorithm by two numerical examples.
\end{abstract}

\section{INTRODUCTION}

Differential equations arise from various practical problems in mathematics and physics such as gas dynamics, nuclear physics, chemical reaction and geological prospecting etc. Multi-point BVPs can solve the contradictions in the process of actual research status efficiently and enhance their compatibility. Therefore, this problem has received a lot of attention of researchers. In [1-4], the existence and uniqueness are investigated. In [5], the author give the approximate solutions of a certain class of singular two-point (BVPs) by the Sinc-Galerkin method and homotopy-perturbation method. In [6], the author solve two-point BVPs by variational iteration method. In [7], the author present a method for solving a class of singular two-point BVPs based on cubic splines. For more information, please refer to [8-13]. Recently, in [14], the author adopt differential transformation method to solve the two-point BVPs

$$
\left\{\begin{array}{l}
u^{\prime \prime}(x)+\frac{1}{x} u^{\prime}(x)+q(x) u(x)=f(x), 0<x \leq 1 \\
u(0)=a_{1}, u(1)=b_{1}, \text { or } u^{\prime}(0)=a_{2}, u(1)=b_{2} .
\end{array}\right.
$$

Received by the editors October 17, 2013. Revised February 5, 2014. Accepted Feb. 9, 2014 2010 Mathematics Subject Classification. 34K10, 34K28, 34M45, 46E22, 47B32 .

Key words and phrases. singular multi-point BVPs, complex reproducing kernel space, operator equation, uniform convergence.

${ }^{*}$ Corresponding author. 
This method is able to provide an approximate solution of (1.1), while it has several disadvantages. This method is based on Taylor series which requires a high degree of smoothness. It has a local convergence region and the function $f(x)$ are all polynomial functions.

Now, we present a new algorithm to make up the deficiencies in [14]. It can be extended to singular multi-point BVPs. We take the problem

$$
\left\{\begin{array}{l}
u^{\prime \prime}(x)+\frac{1}{p(x)} u^{\prime}(x)+q(x) u(x)=f(x), 0<x \leq 1, \\
u^{\prime}(0)=0, u(1)=\delta u(c),
\end{array}\right.
$$

where $p(x), q(x), f(x)$ are continuous complex functions on $(0,1]$ and $x=0$ is the singular value point of $p(x), c, \delta$ are complex constants and $c \in(0,1), \delta \neq 1$.

In this paper, we construct a complex reproducing kernel space $W_{2}^{3}[0,1]$. Then (1.2) can be transformed into an equivalent operator equation. Its approximate solution is provided. We also analyze the convergence and complexity of this algorithm. Finally, we give some numerical examples to verify the effectiveness of our algorithm.

\section{Several Complex Reproducing Kernel Spaces}

2.1. The complex reproducing kernel space $W_{2}^{3}[0,1]$. We define the inner product space $W_{2}^{3}[0,1]=\left\{u(x) \mid u^{\prime \prime}\right.$ is absolutely continuous complex function, $u^{(3)} \in$ $\left.L^{2}[0,1], u^{\prime}(0)=0, u(1)=\delta u(c)\right\}$.

The inner product is given by

$$
\langle u, v\rangle=u^{\prime \prime}(0) \bar{v}^{\prime \prime}(0)+\int_{0}^{1} u^{\prime \prime \prime}(x) \bar{v}^{\prime \prime \prime}(x) \mathrm{d} x .
$$

Lemma 2.1. The space $W_{2}^{3}[0,1]$ is a complex reproducing kernel space.

The proof can be found in [15]. Next, we give the reproducing kernel function $R_{y}(x)$ of $W_{2}^{3}[0,1]$. For each $y \in[0,1]$ and each $u(x) \in W_{2}^{3}[0,1]$, by applying $(2.1)$, we have

$$
u(y)=\left\langle u(x), R_{y}(x)\right\rangle,
$$

that is

$$
\left\langle u(x), R_{y}(x)\right\rangle=u^{\prime \prime}(0) \bar{R}_{y}^{\prime \prime}(0)+\int_{0}^{1} u^{\prime \prime \prime}(x) \frac{\partial^{3} \bar{R}_{y}(x)}{\partial x^{3}} \mathrm{~d} x
$$

where

$$
\int_{0}^{1} u^{\prime \prime \prime}(x) \frac{\partial^{3} \bar{R}_{y}(x)}{\partial x^{3}} \mathrm{~d} x=\int_{0}^{c} u^{\prime \prime \prime}(x) \frac{\partial^{3} \bar{R}_{y}(x)}{\partial x^{3}} \mathrm{~d} x+\int_{c}^{1} u^{\prime \prime \prime}(x) \frac{\partial^{3} \bar{R}_{y}(x)}{\partial x^{3}} \mathrm{~d} x .
$$


We can obtain

$$
\begin{aligned}
u(y)= & \left\langle u(x), R_{y}(x)\right\rangle=u^{\prime \prime}(0)\left(\frac{\partial^{2} \bar{R}_{y}(0)}{\partial x^{2}}-\frac{\partial^{3} \bar{R}_{y}(0)}{\partial x^{3}}\right)+\sum_{i=1}^{2}(-1)^{i} u^{(i)}(1) \frac{\partial^{(5-i)} \bar{R}_{y}(1)}{\partial x^{(5-i)}} \\
& -u(0) \frac{\partial^{5} \bar{R}_{y}(0)}{\partial x^{5}}+\sum_{i=1}^{2}(-1)^{i} u^{(i)}(c)\left(\frac{\partial^{(5-i)} \bar{R}_{y}(c-)}{\partial x^{(5-i)}}-\frac{\partial^{(5-i)} \bar{R}_{y}(c+)}{\partial x^{(5-i)}}\right) \\
& -\int_{0}^{c} u(x) \frac{\partial^{6} \bar{R}_{y}(x)}{\partial x^{6}} \mathrm{~d} x+u(c)\left(\frac{\partial^{5} \bar{R}_{y}(c-)}{\partial x^{5}}-\frac{\partial^{5} \bar{R}_{y}(c+)}{\partial x^{5}}+\delta \frac{\partial^{5} \bar{R}_{y}(1)}{\partial x^{5}}\right) \\
& -\int_{c}^{1} u(x) \frac{\partial^{6} \bar{R}_{y}(x)}{\partial x^{6}} \mathrm{~d} x .
\end{aligned}
$$

Now, we have

$$
\left\{\begin{array}{l}
\frac{\partial^{2} \bar{R}_{y}(0)}{\partial x^{2}}-\frac{\partial^{3} \bar{R}_{y}(0)}{\partial x^{3}}=0 \\
\frac{\partial^{(5-i)} \bar{R}_{y}(1)}{\frac{\partial x^{(5-i)}}{R}{ }^{5}(0)}=0, i=1,2 \\
\frac{\partial^{5} x^{5}}{\partial x^{5} \bar{R}_{y}(c-)}=0 \\
\frac{\partial^{(5-i)}}{x^{(5-i)}}-\frac{\partial^{(5-i)} \bar{R}_{y}(c+)}{\partial x^{(5-i)}}=0, i=1,2, \\
\frac{\partial^{5} x_{y}(c-)}{\partial x^{5}}-\frac{\partial^{5} \bar{R}_{y}(c+)}{\partial x^{5}}+\delta \frac{\partial^{5} \bar{R}_{y}(1)}{\partial x^{5}}=0 .
\end{array}\right.
$$

Since $R_{y}(x) \in C^{2}$, we get

$$
\left\{\begin{array}{l}
\frac{\partial^{i} \bar{R}_{y}(c-)}{\partial x^{i}}=\frac{\partial^{i} \bar{R}_{y}(c+)}{\partial x^{i}}, \\
\frac{\partial^{i} \bar{R}_{y}(y-)}{\partial x^{i}}=\frac{\partial^{i} \bar{R}_{y}(y+)}{\partial x^{i}} \cdot(i=0,1,2)
\end{array}\right.
$$

Similarly, if $0 \leq y \leq c$, the function $R_{y}(x)$ should satisfy the differential equation:

$$
\frac{\partial^{6} \bar{R}_{y}(x)}{\partial x^{6}}=\left\{\begin{array}{cc}
-\delta(x-y), & 0 \leq x \leq c \\
0, & c<x \leq 1
\end{array}\right.
$$

and if $c \leq y \leq 1$, the function $R_{y}(x)$ should satisfy the differential equation:

$$
\frac{\partial^{6} \bar{R}_{y}(x)}{\partial x^{6}}=\left\{\begin{array}{cc}
0, & 0 \leq x \leq c \\
-\delta(x-y), & c<x \leq 1
\end{array}\right.
$$

If $x \neq y,(2.5)$ and $(2.6)$ become $\frac{\partial^{6} \bar{R}_{y}(x)}{\partial x^{6}}=0$. Its characteristic equation is

$$
\lambda^{6}=0
$$


the characteristic roots are $\lambda_{i}=0,(i=1,2, \cdots, 6)$. So, we assume that

$$
R_{y}(x)=\left\{\begin{array}{l}
R 1(x, y)=c_{11}+c_{12} x+c_{13} x^{2}+c_{14} x^{3}+c_{15} x^{4}+c_{16} x^{5}, y<x<c, \\
R 2(x, y)=c_{21}+c_{22} x+c_{23} x^{2}+c_{24} x^{3}+c_{25} x^{4}+c_{26} x^{5}, y<c<x, \\
R 3(x, y)=c_{31}+c_{32} x+c_{33} x^{2}+c_{34} x^{3}+c_{35} x^{4}+c_{36} x^{5}, c<y<x, \\
R 4(x, y)=c_{41}+c_{42} x+c_{43} x^{2}+c_{44} x^{3}+c_{45} x^{4}+c_{46} x^{5}, c<x<y, \\
R 5(x, y)=c_{51}+c_{52} x+c_{53} x^{2}+c_{54} x^{3}+c_{55} x^{4}+c_{56} x^{5}, x<c<y, \\
R 6(x, y)=c_{61}+c_{62} x+c_{63} x^{2}+c_{64} x^{3}+c_{65} x^{4}+c_{66} x^{5}, x<y<c .
\end{array}\right.
$$

Since

$$
\frac{\partial^{6} \bar{R}_{y}(x)}{\partial x^{6}}=-\delta(x-y),
$$

we get

$$
\frac{\partial^{i} \bar{R}_{y}(y-)}{\partial x^{i}}=\frac{\partial^{i} \bar{R}_{y}(y+)}{\partial x^{i}},(i=3,4)
$$

and

$$
\frac{\partial^{5} \bar{R}_{y}(y-)}{\partial x^{5}}=1+\frac{\partial^{5} \bar{R}_{y}(y+)}{\partial x^{5}} .
$$

In addition, we also need

$$
R_{y}^{\prime}(0)=0, \quad R_{y}(1)=\delta R_{y}(c) .
$$

Combining(2.3), (2.4), (2.8) - (2.10) as well as $y \in(0, c)$ and $y \in(c, 1)$, we get 36 equations. We can find the undetermined coefficients $c_{i j}$ of $(2.7)$ by solving the equations. If $c=1 / 2$ and $\delta=1+2 i, R_{y}(x)$ is the following expression

$$
R_{y}(x)=\left\{\begin{array}{rlrl}
R 1(x, y)= & \frac{1}{3840}\left[-(80+120 i) x^{3}+(80+40 i) x^{4}-32 x^{5}-160 x y^{4}\right. \\
& +40 x^{2}\left(-(6+9 i)+24 y^{2}+8 y^{3}\right)+5\left(47-(48-72 i) y^{2}\right. \\
& \left.\left.-(16-24 i) y^{3}+(16-8 i) y^{4}\right)\right], \quad y<x<c, \\
R 2(x, y)= & \frac{1}{7680}\left[32 i x^{3}\left(5 x-10-x^{2}\right)+40 x^{2}\left(48 y^{2}-(14+17 i)+16 y^{3}\right)\right. \\
& -10 x\left((i-2)+32 y^{4}\right)+(2-i)\left(187+94 i-(336-192 i) y^{2}\right. \\
& \left.\left.-(112-64 i) y^{3}+80 y^{4}\right)\right], \quad y<c<, \\
R 3(x, y)= & \frac{1}{3840}\left(233-160 i x^{3}+80 i x^{4}-16 i x^{5}+(10+5 i) y-(280\right. \\
& -340 i) y^{2}+160 i y^{3}-80 i y^{4}+(32+16 i) y^{5}+20 x^{2}(-(14+17 i) \\
& \left.\left.+48 y^{2}+16 y^{3}\right)-5 x\left((i-2)+32 y^{4}\right)\right), & c<y<x, \\
R 4(x, y)= & \overline{R 3(y, x)}, & & c<x<y, \\
R 5(x, y)= & \overline{R 2(y, x)}, & x<c<y, \\
R 6(x, y)= & \overline{R 1(y, x),} & & x<c .
\end{array}\right.
$$


2.2. The complex reproducing kernel space $W_{2}^{1}[0,1] . W_{2}^{1}[0,1]=\{u(x) \mid u$ is absolutely continuous complex function, $\left.u^{\prime} \in L^{2}[0,1]\right\}$.

The inner product is given by

$$
\langle u, v\rangle=u(0) \bar{v}(0)+\int_{0}^{1} u^{\prime}(x) \bar{v}^{\prime}(x) \mathrm{d} x .
$$

It is easy to prove that $W_{2}^{1}[0,1]$ is a complex reproducing kernel space and its reproducing kernel is

$$
K_{y}(x)= \begin{cases}1+x, & x \leq y \\ 1+y, & y<x\end{cases}
$$

\section{A Solution of (1.2)}

In this section, we investigate how to obtain approximate solutions of (1.2). First, we transform (1.2) into an equivalent operator equation (3.1). Then we give its approximate solution. Also, the convergence and complexity analysis are provided.

3.1. Equivalent operator equation. The equation (1.2) can be transformed into the following form:

$$
\left\{\begin{array}{l}
x^{\alpha} u^{\prime \prime}(x)+\frac{x^{a}}{p(x)} u^{\prime}(x)+x^{a} q(x) u(x)=x^{a} f(x), 0<x \leq 1, \\
u^{\prime}(0)=0, u(1)=\delta u(c)
\end{array}\right.
$$

where $\alpha$ is constant and satisfy $\lim _{x \rightarrow 0} \frac{x^{a}}{p(x)}=b \quad(b \neq 0)$.

Define linear operator $\mathbb{L}: W_{2}^{3}[0,1] \longrightarrow W_{2}^{1}[0,1]$ by

$$
\mathbb{L} u=x^{\alpha} u^{\prime \prime}(x)+\frac{x^{a}}{p(x)} u^{\prime}(x)+x^{a} q(x) u(x) .
$$

Obviously, operator $\mathbb{L}$ is bounded. The equation (1.2) can be converted into an equivalent operator equation:

$$
\mathbb{L} u=f_{1}(x),
$$

where $f_{1}(x)=x^{a} f(x)$.

3.2. The numerical solution for operator equation (3.1). We choose a countable dense subset $\left\{x_{i}\right\}_{i=1}^{\infty} \subset(0,1]$ and define $\psi_{i}(x)$ as

$$
\psi_{i}(x) \stackrel{\text { def }}{=}\left(\mathbb{L} R_{x}(\cdot)\right)\left(x_{i}\right) \in W_{2}^{3}[0,1] .
$$


Theorem 3.1. The function system $\left\{\psi_{i}(x)\right\}_{i=1}^{\infty}$ is a complete system in the space $W_{2}^{3}[0,1]$.

Proof. For an arbitrary $i$, we have,

$$
\begin{aligned}
0 & =\left\langle u(x), \psi_{i}(x)\right\rangle=\left\langle u(x),\left(\mathbb{L} R_{x}(\cdot)\right)\left(x_{i}\right)\right\rangle=\mathbb{L}\left(\left\langle u(x), R_{x}(\cdot)\right\rangle\right)\left(x_{i}\right) \\
& =\mathbb{L}(u(\cdot))\left(x_{i}\right)=(\mathbb{L} u)\left(x_{i}\right) .
\end{aligned}
$$

Note that $\left\{x_{i}\right\}_{i=1}^{\infty}$ is dense in $[0,1]$, so $(\mathbb{L} u)(x)=0$. By the existence of $\mathbb{L}^{-1}$, it follows that $u \equiv 0$. Therefore, $\left\{\psi_{i}(x)\right\}_{i=1}^{\infty}$ is a complete system in $W_{2}^{3}[0,1]$.

Furthermore, we obtain an orthogonal system $\left\{\widetilde{\psi}_{i}(x)\right\}_{i=1}^{\infty}$ of $W_{2}^{3}[0,1]$ derived from Gram-Schmidt orthonormalization process from $\left\{\psi_{i}(x)\right\}_{i=1}^{\infty}$ :

$$
\widetilde{\psi}_{i}(x)=\sum_{k=1}^{i} \beta_{i k} \psi_{k}(x) .
$$

Theorem 3.2. If $\left\{x_{i}\right\}_{i=1}^{\infty}$ is dense on $[0,1]$, then the solution of $(1.2)$ is

$$
u(x)=\sum_{i=1}^{\infty} \sum_{k=1}^{i} \bar{\beta}_{i k} f_{1}\left(x_{k}\right) \widetilde{\psi}_{i}(x) .
$$

Proof. We expand $u(x)$ into a Fourier series as follows

$$
\begin{aligned}
u(x) & =\sum_{i=1}^{\infty}\left\langle u(x), \widetilde{\psi}_{i}(x)\right\rangle \widetilde{\psi}_{i}(x)=\sum_{i=1}^{\infty}\left\langle u(x), \sum_{k=1}^{i} \beta_{i k} \psi_{k}(x)\right\rangle \widetilde{\psi}_{i}(x) \\
& =\sum_{i=1}^{\infty} \sum_{k=1}^{i} \bar{\beta}_{i k}\left\langle u(x), \psi_{k}(x)\right\rangle \widetilde{\psi}_{i}(x)=\sum_{i=1}^{\infty} \sum_{k=1}^{i} \bar{\beta}_{i k}\left\langle u(x),\left(\mathbb{L} R_{x}(\cdot)\right)\left(x_{k}\right)\right\rangle \widetilde{\psi}_{i}(x) \\
& =\sum_{i=1}^{\infty} \sum_{k=1}^{i} \bar{\beta}_{i k} \mathbb{L}\left(\left\langle u(x),\left(R_{x}(\cdot)\right)\right\rangle\right)\left(x_{k}\right) \widetilde{\psi}_{i}(x)=\sum_{i=1}^{\infty} \sum_{k=1}^{i} \bar{\beta}_{i k} f_{1}\left(x_{k}\right) \widetilde{\psi}_{i}(x)
\end{aligned}
$$

Now, we can get the approximate solution $u_{n}(x)$ by truncating the $n t h-t e r m$ of the exact solution $u(x)$,

$$
u_{n}(x)=\sum_{i=1}^{n} \sum_{k=1}^{i} \bar{\beta}_{i k} f_{1}\left(x_{k}\right) \widetilde{\psi}_{i}(x) .
$$




\subsection{Theoretical analysis for our algorithm.}

Theorem 3.3. An approximate solution $u_{n}(x)$ is uniform convergence to $u(x)$ on $[0,1]$. Moreover, $u_{n}^{\prime}(x), u_{n}^{\prime \prime}(x)$ are both uniform convergence to $u^{\prime}(x)$ and $u^{\prime \prime}(x)$ on $[0,1]$.

Proof. Note that

$$
u_{n}(x)=\left\langle u_{n}, R_{x}\right\rangle, u(x)=\left\langle u, R_{x}\right\rangle
$$

and

$$
u_{n}^{(i)}(x)=\left\langle u_{n}, \partial_{x}^{i} R_{x}\right\rangle, u^{(i)}(x)=\left\langle u, \partial_{x}^{i} R_{x}\right\rangle \cdot(i=1,2)
$$

By applying Schwarz's inequality and the boundedness of $\left\|\partial_{x}^{i} R_{x}\right\|(i=0,1,2)$, we have

$$
\left|u_{n}^{(i)}(x)-u^{(i)}(x)\right| \leq\left\|u_{n}-u\right\|\left\|\partial_{x}^{i} R_{x}\right\| \leq M_{i}\left\|u_{n}-u\right\| \rightarrow 0 .
$$

So

$$
u_{n}^{(i)}(x) \rightrightarrows u^{(i)}(x) \quad(i=0,1,2)
$$

Theorem 3.4. The time complexity of the algorithm is $O\left(n^{3}\right)$.

Proof. There are three steps to calculate the approximate solution $u_{n}(x)$ of (1.2) .

(1) Assume the number of multiplications required is $C$ in one calculation of the inner product $\left\langle\varphi_{i}, \varphi_{j}\right\rangle$, then the total number of multiplications required is $n(n+1) C / 2$ in calculation of all inner products.

(2) Orthogonalization of the system $\left\{\psi_{i}(x)\right\}_{i=1}^{n}$ needs 3 layers of nested loops, that is, the number of multiplication is

$$
\sum_{i=1}^{n} \sum_{k=1}^{i} \sum_{j=1}^{k}=\frac{n(n+1)(n+2)}{6} .
$$

(3) The number of multiplication is $n^{2}$ when calculating $u_{n}(x)$ using (3.2).

To sum up, the total number of multiplication is

$$
\frac{n(n+1) C}{2}+\frac{n(n+1)(n+2)}{6}+n^{2}=O\left(n^{3}\right) .
$$




\section{Numerical EXAmples}

In this section, some numerical examples are studied to demonstrate the accuracy of the present algorithm. Results obtained by this algorithm are compared with the exact solution of each example and are shown to be in good agreement with the exact solution.

Example 1. Consider equation

$$
\left\{\begin{array}{l}
u^{\prime \prime}(x)+\frac{1}{\sin x+i x} u^{\prime}(x)+i u(x)=f(x), 0<x \leq 1 \\
u^{\prime}(0)=0, u(1)=(1+2 i) u\left(\frac{1}{2}\right)
\end{array}\right.
$$

where $f(x)=\frac{3+2 i}{13}\left[(8+4 i)-(16-8 i) \sqrt{e}-8 i e-(1-5 i) e^{x}+(3-2 i) x-((2-4 i)+\right.$ $\left.(4+8 i) \sqrt{e}-4 e) x^{2}+\frac{(-3+2 i)+(3-2 i) e^{x}+(4-8 i) x+(8+16 i) \sqrt{e} x-8 e x}{x-i \sin x}\right]$. Its exact solution is $u(x)=\frac{6+4 i}{13}\left[((1-2 i)+(2+4 i) \sqrt{e}-2 e) x^{2}-e^{x}+x\right] i$. Applying our algorithm and taking the number of nodes as $n=50$ and 100, the absolute errors of real part (a.e.Re) and the absolute errors of imaginary part (a.e.Im) are shown in Table 1. It shows that the approximate solution is getting more and more accurate as $\mathrm{n}$ increases.

Table1: The absolute errors for Example 1

\begin{tabular}{lllll}
\hline $\mathrm{x}$ & a.e.Re$(\mathrm{n}=50)$ & a.e.Im(n=50) & a.e.Re $(\mathrm{n}=100)$ & a.e.Im $(\mathrm{n}=100)$ \\
\hline 0.1 & $4.2040 \mathrm{E}-5$ & $3.9676 \mathrm{E}-5$ & $9.5771 \mathrm{E}-6$ & $5.2571 \mathrm{E}-6$ \\
0.2 & $4.2197 \mathrm{E}-5$ & $1.3767 \mathrm{E}-5$ & $8.5274 \mathrm{E}-6$ & $2.7894 \mathrm{E}-7$ \\
0.3 & $3.3794 \mathrm{E}-5$ & $6.1748 \mathrm{E}-7$ & $3.3151 \mathrm{E}-6$ & $1.5179 \mathrm{E}-6$ \\
0.4 & $2.5060 \mathrm{E}-5$ & $7.6275 \mathrm{E}-6$ & $4.3567 \mathrm{E}-6$ & $2.3956 \mathrm{E}-6$ \\
0.5 & $1.6794 \mathrm{E}-5$ & $1.3384 \mathrm{E}-5$ & $2.6555 \mathrm{E}-6$ & $2.8710 \mathrm{E}-6$ \\
0.6 & $9.0774 \mathrm{E}-6$ & $1.7845 \mathrm{E}-5$ & $1.1549 \mathrm{E}-6$ & $3.1825 \mathrm{E}-6$ \\
0.7 & $1.8277 \mathrm{E}-6$ & $2.1730 \mathrm{E}-5$ & $2.0479 \mathrm{E}-7$ & $3.4707 \mathrm{E}-6$ \\
0.8 & $5.0567 \mathrm{E}-6$ & $2.5501 \mathrm{E}-5$ & $1.4713 \mathrm{E}-6$ & $3.8216 \mathrm{E}-6$ \\
0.9 & $1.1695 \mathrm{E}-5$ & $2.9506 \mathrm{E}-5$ & $2.6878 \mathrm{E}-6$ & $4.3009 \mathrm{E}-6$ \\
1.0 & $1.8220 \mathrm{E}-5$ & $3.4027 \mathrm{E}-5$ & $3.8960 \mathrm{E}-6$ & $4.9617 \mathrm{E}-6$ \\
\hline
\end{tabular}

Example 2. Consider equation

$$
\left\{\begin{array}{l}
u^{\prime \prime}(x)+\frac{1}{\sin x} u^{\prime}(x)+u(x)=f(x), 0<x \leq 1, \\
u^{\prime}(0)=0, u(1)=0
\end{array}\right.
$$


where

$$
f(x)=\left\{\begin{array}{l}
x-\frac{11}{2}, 0<x<\frac{1}{2}, \\
-5+\sqrt{\left(x-\frac{1}{2}\right)}, \frac{1}{2} \leq x \leq 1 .
\end{array}\right.
$$

The function $f(x)$ is continuous at $x=\frac{1}{2}$, but not differentiable. So the method of [14] is invalid for example 2. While using our algorithm, we choose 100 points in $(0,1]$. The numerical results $\left|\mathbb{L} u_{100}-f\right|$ are given in the following Table 2.

\begin{tabular}{|c|c|c|c|}
\hline $\mathrm{x}$ & $\left|\mathbb{L} u_{100}-f\right|$ & $\mathrm{x}$ & $\left|\mathbb{L} u_{100}-f\right|$ \\
\hline$\overline{0.05}$ & $7.1054 \mathrm{E}-15$ & 0.55 & $4.4408 \mathrm{E}-14$ \\
\hline 0.1 & $9.7699 \mathrm{E}-15$ & 0.6 & $1.9539 \mathrm{E}-14$ \\
\hline 0.15 & $6.2172 \mathrm{E}-15$ & 0.65 & $1.2434 \mathrm{E}-14$ \\
\hline 0.2 & $1.5987 \mathrm{E}-14$ & 0.7 & $5.3290 \mathrm{E}-15$ \\
\hline 0.25 & $1.5099 \mathrm{E}-14$ & 0.75 & $3.1086 \mathrm{E}-14$ \\
\hline 0.3 & 2.3092E-14 & 0.8 & $1.7763 \mathrm{E}-14$ \\
\hline 0.35 & 7.9936E-15 & 0.85 & $1.7763 \mathrm{E}-14$ \\
\hline 0.4 & $2.5757 \mathrm{E}-14$ & 0.9 & $3.3750 \mathrm{E}-14$ \\
\hline 0.45 & $5.3290 \mathrm{E}-15$ & 0.95 & $3.9968 \mathrm{E}-14$ \\
\hline 0.5 & $2.5757 \mathrm{E}-14$ & 1.0 & $1.7763 \mathrm{E}-14$ \\
\hline
\end{tabular}

\section{Conclusion}

In this paper, we present a new numerical algorithm in complex reproducing kernel space for singular multi-point BVPs. We give the rigorous theoretical analysis, the uniform convergence of the approximate solution. The numerical examples show that by using this algorithm we obtain better solution and fix the deficiencies of [14].

\section{REFERENCES}

1. F. Wong \& W. Lian: Positice solution for singular boundary value problems. Comput. Math. Appl. 32 (1996), no. 9, 41-49.

2. P. Kelevedjiev: Existence of positice solution to a singular second order boundary value problem. Nonlinear Anal. 50 (2002), 1107-1118.

3. X. Xu \& J. Ma: A note on singular nonlinear boundary value problems. J. Math. Anal. Appl. 293 (2004), 108-124.

4. Y. Liu \& H. Yu: Existence and uniqueness of positice solution for singular boundary value problem. Comput. Math. Appl. 50 (2005), 133-143.

5. K. Al-Khaled: Theory and computation in singular boundary value problems. Chaos Solitons Fractals. 33 (2007), 678-684. 
6. L.U. Junfeng: Variational iteration method for solving two-point boundary value problems. J. Comput. Appl. Math. 207 (2007), 92-101.

7. A.S.V. Ravi Kanth \& Y.N. Reddy: Cubic spline for a class of two-point boundary value problems. Appl. Math. Comput. 207 (2005), 733-740.

8. John R. Graef \& Lingju Kong: Necessary and sufficient conditions for the existence of symmetric positive solutions of multi-point boundary value problems. Nonlinear Anal. 68 (2008), 1529-1552.

9. Qingkai Kong \& Min Wang: Positive solutions of even order system periodic boundary value problems. Nonlinear Anal. 72 (2010), 1778-1791.

10. Siraj-ul-Islam, Ikram A \& Tirmizi: Nonpolynomial spline approach to the solution of a system of second-order boundary-value problems. Appl. Math. Comput. 173 (2006), 1208-1218.

11. Siraj-ul-Islam, Imran Aziz \& Booidararler: The numerical solution of second-order boundary-value problems by collocation method with the Haar wavelets. Math. Comput. Modelling. 52 (2010), 1577-1590.

12. J. Caballero, J. Harjani \& K. Sadarangani: Positive solutions for a class of singular fractional boundary value problems. Comput. Math. Appl. 62 (2011), 1325-1332.

13. Tieshan He, Fengjian Yang, Chuanyong Chen \& Shiguo Peng: Existence and multiplicity of positive solutions for nonlinear boundary value problems with a parameter. Comput. Math. Appl. 61 (2011), 3355-3363.

14. A.S.V. Ravi Kanth \& K.Aruna: Solution of singular two-point boundary value problems using differential transformation method. Phys. Lett. A. 372 (2008), 4671-4673

15. Boying Wu \& Yingzhen Lin: Application of the reproducing kernel space. Science Press, 2012.

${ }^{a}$ School of Science, Zhuhai Campus, Beijing Institute of Technology, Zhuhai GuangDONG , 519085

Email address: jyt-002@126.com

${ }^{\mathrm{b}}$ School of Science, Zhuhai Campus, Beijing Institute of Technology, Zhuhai GuangDONG , 519085

Email address: liliy5501@gmail.com 